\title{
Volumetric Object Reconstruction in Multi-Camera Scenarios
}

\author{
Enrique Hernández-Murillo, Rosario Aragües, Gonzalo López-Nicolás \\ Grupo de Robótica Percepción y Tiempo Real \\ Instituto de Investigación en Ingeniería de Aragón (I3A) \\ Universidad de Zaragoza, Mariano Esquillor s/n, 50018, Zaragoza, Spain. \\ Tel. +34-976762707, e-mail: ehernandez@unizar.es
}

\begin{abstract}
Volumetric reconstruction of unknown objects is essential in robotic manipulation. Building the 3D model requires a set of views so we consider a multicamera scenario. We study an effective configuration strategy to address camera constraints such as the limited field of view or self-occlusions.
\end{abstract}

\section{Introduction}

Nowadays in manufacturing industry, perception is one of the most basic tasks related to manipulation of objects. Here, the main goal is the reconstruction of an, a priori, unknown object. On the one hand, active vision could be taken as a viable alternative. This technique may require using a vision-based sensor, mounted on a mobile robot, providing dense 3D input data. Active vision methods address the problem of generating a complete volumetric model of the object, as fast as possible, by solving the next best view problem. Thus, they compute a sequence of camera locations (position and orientation) around the object. To reduce the number of candidate views, they may be restricted to a sphere or a cylinder around the object. However, one of the main challenges involved in active vision are deformable or mobile objects, which requires an instantaneous perception of the object. The main drawback of active vision in this framework is the time required to follow the sequence of camera locations. So, how can be something perceived from multiple views at once? We believe the answer could be to fuse the information from several cameras in a volumetric map of the entire object at each moment. Besides, the multi-camera system could be a good choice if the model is deformable or if the object is mobile. We place several cameras in a geometric formation around the moving object to enclose it and track it. This strategy [4] ensures that the multi-robot system can perform full perception of the target along its motion.

In this work, we consider the problem of the reconstruction of an a priori unknown object that deforms and moves (its boundaries are not limited).
We approach the problem of the instantaneous 3D reconstruction using a probabilistic volumetric map built in real time. Besides, we extend the problem to a multi-robot team scenario. We propose and evaluate a multi-camera generic system architecture for the camera positioning mechanism to model 3D arbitrary objects. The multi-robot scenario affords us to elaborate a strategy which allows not losing the tracking of the object when it is in movement.

\section{Multi-camera system architecture}

We divide the multi-robot reconstruction task in three modules (i) instantaneous volumetric model reconstruction, (ii) multi-camera view planner (Strategy) and (iii) multi-camera positioning mechanism. In this section we present a general overview of our system. Its structure is built upon the framework observed in [3]. Conceptually, it is a modular system architecture that consists of different independent modules interacting through communication interfaces, as depicted in Fig. 2.

- The Perception System is responsible for data acquisition and its processing. The main components that build this system are the sensor module and the $3 D$ perception Module. In the multi-camera scenario, the output is as many point-clouds of observed 3D points as the number of cameras we have.

- The World Representation Module registers all the data perceived within the map and gives access to the current map. This module also carries out the reconstruction task with OctoMap [2].

- The Motion Planning and Control implements the camera positioning mechanism that locates the robot, and the Robot Interface that provides the interface between View Planner and the robot.

- The View Planner Module carries out path planning and sends the next viewpoint to the robot, which receives it using the Robot 
Interface. This new view is where the robot must move the sensor.

\section{Experiments}

The simulation of the reconstruction scene includes an object placed on an empty world (Fig. 1). The model used is available on-line: the Stanford bunny (Fig. 1). Each robot is a free-flying stereo camera with 6 DoF. Although the camera can move freely, the strategy implemented [4] assumes unicycle kinematics. The simulation is carried out in Gazebo, this environment the stereo processing can be carried out using ROS (Robot Operating System). The probabilistic volumetric map is based on OctoMap [2], where every pair of stereo-cameras share their computed point-cloud message to be integrated on it. The camera positioning planner is based in [4], which locates the cameras forming a hexagonal formation (Fig. 3).

\section{Conclusions}

In this work, we have developed an active reconstruction framework based on [1] and the extension to a multi-robot scenario. We have divided our approach into three independent modules: volumetric reconstruction, multi-camera extension, and multi-camera strategy. Given the good results obtained in simulation, our future work will focus in several improvements of the system framework, such as testing different multi-robot strategies or the optimization of the communication interface between the components.

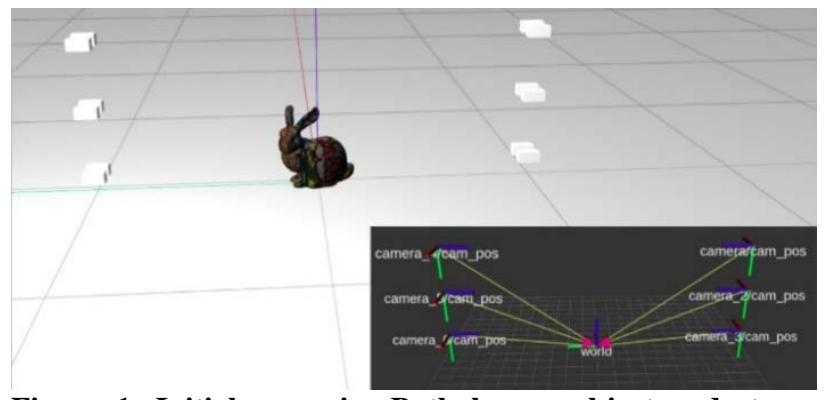

Figure 1. Initial scenario: Both bunny object and stereo camera pairs are simulated in Gazebo and shown by Rviz.

\section{Acknowledgements}

This work was supported by projects COMMANDIA SOE2/P1/F0638 (Interreg Sudoe Programme, ERDF) and PGC2018-098719-B-I00 (MCIU/AEI/FEDER, UE).

\section{REFERENCIAS}

[1]. DELMERICO, J., ISLER, S., SABZEVARI, R., and SCARAMUZZA, D. (2018). A comparison of volumetric information gain metrics for active 3D object reconstruction. Autonomous Robots.

[2]. HORNUNG, A., WURM, K. M., BENNEWITZ, M., STACHNISS, C., and BURGARD, W. (2013). OctoMap: An efficient probabilistic 3D mapping framework based on octrees. Autonomous Robots.

[3]. ISLER, S., SABZEVARI, R., DELMERICO, J., and SCARAMUZZA, D. (2016). An information gain formulation for active volumetric 3D reconstruction. In IEEE international conference on robotics and automation (ICRA).

[4]. LÓPEZ-NICOLÁS, G., ARANDA, M., and MEZOUAR, Y. (2017). Formation of differentialdrive vehicles with field-of-view constraints for enclosing a moving target. In IEEE international conference on robotics and automation, pp. 261-266.

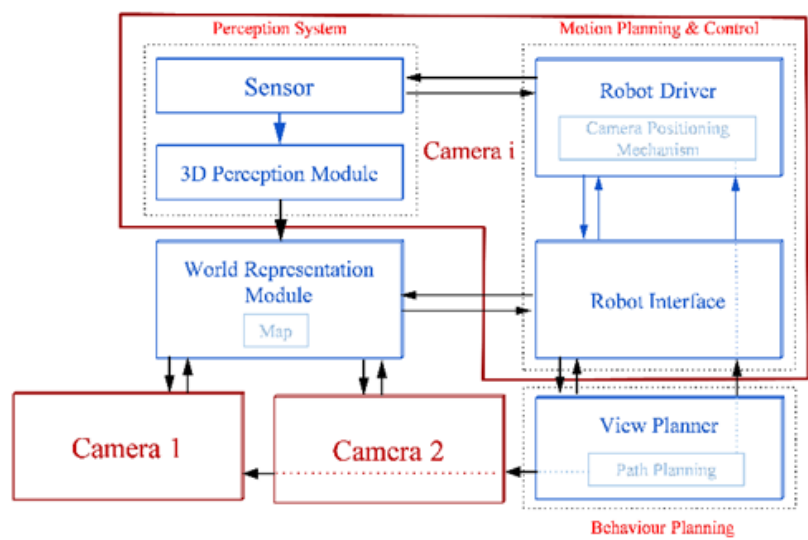

Figure 2. Conceptual system overview: Main modules, and their communication interfaces (arrows) are shown.

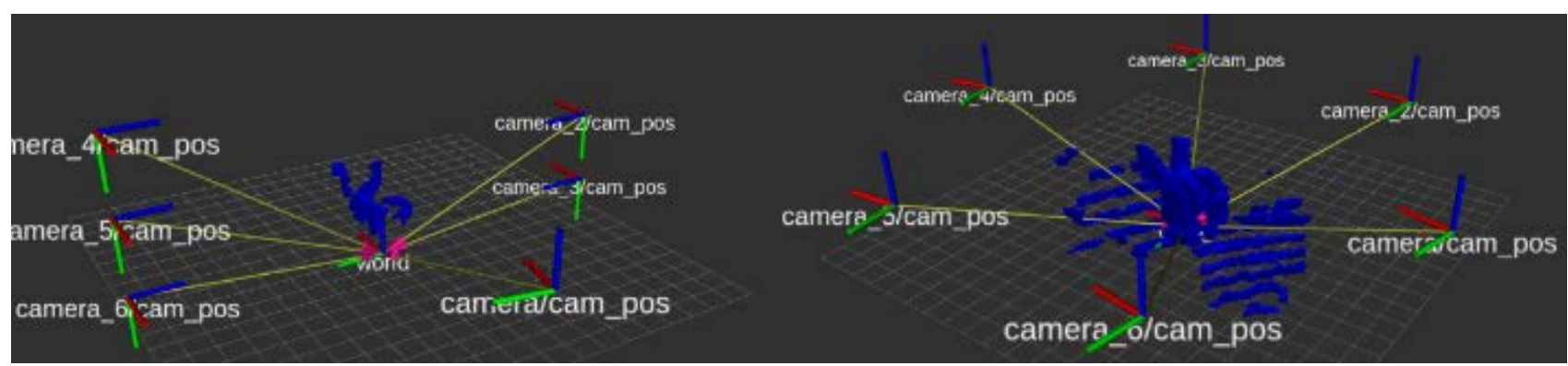

Figure 3. Simulation reconstruction scene: The model of the bunny object and the multi-camera strategy are visualized.

Revista “Jornada de Jóvenes Investigadores del I3A”, vol. 7 (Actas de la VIII Jornada de Jóvenes Investigadores del I3A - 6 de junio de 2019). ISSN 2341-4790. 